\title{
Somatic mutations and increased lymphangiogenesis observed in a rare case of intramucosal gastric carcinoma with lymph node metastasis
}

\author{
Naoki Ikari ${ }^{1,2, *}$, Shota Aoyama ${ }^{1, *}$, Akiyoshi Seshimo ${ }^{1}$, Yuji Suehiro ${ }^{3}$, Tomoko \\ Motohashi $^{3}$, Shohei Mitani ${ }^{3}$, Sawako Yoshina ${ }^{3}$, Etsuko Tanji ${ }^{2}$, Akiko Serizawa ${ }^{1}$, \\ Takuji Yamada ${ }^{1}$, Kiyoaki Taniguchi ${ }^{1}$, Masakazu Yamamoto ${ }^{1}$ and Toru Furukawa ${ }^{2,4,5}$ \\ ${ }^{1}$ Department of Surgery, Institute of Gastroenterology, Tokyo Women's Medical University, Tokyo, Japan \\ ${ }^{2}$ Institute for Integrated Medical Sciences, Tokyo Women's Medical University, Tokyo, Japan \\ ${ }^{3}$ Department of Physiology, Tokyo Women's Medical University School of Medicine, Tokyo, Japan \\ ${ }^{4}$ Department of Surgical Pathology, Tokyo Women's Medical University Hospital, Tokyo, Japan \\ ${ }^{5}$ Department of Histopathology, Tohoku University Graduate School of Medicine, Sendai, Japan \\ *These authors have contributed equally to this work \\ Correspondence to: Toru Furukawa, email: toru.furukawa@med.tohoku.ac.jp \\ Keywords: early gastric cancer; lymph node metastasis; lymphangiogenesis; NBN; PAX8 \\ Received: May 18, $2017 \quad$ Accepted: January 13, $2018 \quad$ Published: January 22, 2018 \\ Copyright: Ikari et al. This is an open-access article distributed under the terms of the Creative Commons Attribution License 3.0 \\ (CC BY 3.0), which permits unrestricted use, distribution, and reproduction in any medium, provided the original author and source \\ are credited.
}

\section{ABSTRACT}

Background and aim: Intramucosal gastric adenocarcinoma of the wellmoderately differentiated type only exhibits lymph node metastasis in extremely rare cases. We encountered such case and investigated both the lymphangiogenic properties and somatic mutations in the cancer to understand the prometastatic features of early-stage gastric cancer.

Methods: We quantitatively measured the density of lymphatic vessels and identified mutations in $\mathbf{4 1 2}$ cancer-associated genes through next-generation target resequencing of DNA extracted from tumor cells in a formalin-fixed and paraffinembedded tissue. Functional consequence of the identified mutation was examined in vitro by means of gene transfection, immunoblot, and the quantitative real-time polymerase chain reaction assay.

Results: The intramucosal carcinoma was accompanied by abundant lymphatic vessels. The metastatic tumor harbored somatic mutations in NBN, p.P6S, and PAX8, p.R49H. The $P A X 8^{\mathrm{R} 49 \mathrm{H}}$ showed significantly higher transactivation activity toward $E 2 F 1$ than the wild-type PAX8 $(P<0.001)$.

Conclusions: Our data suggest that increased lymphangiogenesis and somatic mutations of NBN and/or PAX8 could facilitate lymph node metastasis from an intramucosal gastric carcinoma. These findings may potentially inform evaluations of the risk of developing lymph node metastasis in patients with intramucosal gastric cancer.

\section{INTRODUCTION}

An endoscopic resection is recommended as a standard treatment (absolute indication) for early gastric carcinomas that fulfill the following criteria: a differentiated-type adenocarcinoma without ulcerative findings, of which the depth of invasion is clinically diagnosed as cT1a and the diameter is $\leq 2 \mathrm{~cm} \mathrm{[1].} \mathrm{This}$ recommendation is based on the rare occurrence of lymph node (LN) metastasis, which is reported to be $0.12 \%(3 / 2402)$ or $0 \%(0 / 6456)$, in patients with early gastric cancer fulfilling the above criteria [1-3]. Despite the rare occurrence of LN metastasis, published reports have emphasized the importance of careful evaluation of 
LN state by using computed tomography (CT) because if a LN metastasis is found, a surgical resection with lymphadenectomy should be performed instead of the endoscopic resection [2, 4]. Hence, mechanistic insights into LN metastasis from early gastric cancer could provide clues for improving the present criteria for the absolute indication of endoscopic resection for early gastric cancer. We obtained a rare opportunity to explore a case of intramucosal gastric adenocarcinoma with synchronized LN metastasis. In this case, we performed a quantitative lymphatic vessel density evaluation, targeted resequencing of 412 cancer-associated genes by next-generation sequencing technology, and a subsequent functional analysis for a mutated gene.

\section{RESULTS}

\section{Patient characteristics and clinical course}

A 68-year-old woman, with a history of eradicated Helicobacter pylori infection, suffered from transient epigastralgia. The patient underwent upper gastrointestinal endoscopy, which elucidated an irregular mucosal lesion in the gastric angle (Figure 1A). A biopsy revealed a tubular adenocarcinoma of the well-differentiated type. Carcinoembryonic antigen (CEA) and carbohydrate antigen 19-9 (CA19-9) levels were $8.5 \mathrm{ng} / \mathrm{ml}$ and $50 \mathrm{U} / \mathrm{ml}$, respectively. Although the lesion seemed to be confined within the gastric mucosa and to fulfill the absolute indication of endoscopic resection, a suspicious LN metastatic lesion (23 mm diameter) beside the left gastric artery was noted on a CT scan (Figure 1B). A laparoscopic tumor biopsy revealed that the tumor was an enlarged LN with tubular adenocarcinoma that was histologically similar to the gastric tumor (Figure 1C). Positron emission tomography/CT showed no apparent uptake except in the LN tumor. Thus, the patient was diagnosed to have an early gastric cancer with a regional LN metastasis and underwent the standard distal gastrectomy with D2 lymph node dissection. After surgery, CEA and CA199 levels normalized. The patient underwent no adjuvant chemotherapy and has remained in good health without any signs of recurrence or other malignant tumors for 39 months (most recent follow-up).

\section{Pathological findings}

A pathological examination identified 4 independent lesions, $1-4 \mathrm{~mm}$ in diameter, in close vicinity to each other at the gastric angle (Figure 2A). The lesions consisted of well-differentiated tubular adenocarcinomas confined within the mucosal layer without any apparent ulceration (Figure 2B, Supplementary Figure 1). There was no scar formation that could suggest segregation of an originally existing tumor into 4 cancerous lesions. The histological features were identical to those of the metastatic adenocarcinoma in the $\mathrm{LN}$ along the lesser curvature (Figure 1C, 2B).

\section{Evaluation of lymphatic vessels}

Formalin-fixed and paraffin-embedded (FFPE) tissue sections were immunohistochemically stained with anti-D2-40 antibody. D2-40-positive lymphatic vessels were particularly dense in the lamina propria of the intramucosal tubular adenocarcinoma in the primary tumor. The dense lymphatic vessels showed irregular shapes with collapsing compared to those in normal mucosa (Figure 2C-2E). Lymphatic vessel density was evaluated semiquantitatively as described by Pak et al [5]. Intratumoral lymphatic vessel density (I-LVD), peritumoral lymphatic vessel density (P-LVD), and control lymphatic vessel density (C-LVD) were $72.3 \pm 4.5,10.7 \pm 3.8$, and $10.7 \pm$ 8.3 , respectively. The I-LVD was significantly higher than $\mathrm{P}-L V D(\mathrm{P}<0.001)$ and C-LVD $(\mathrm{P}<0.001)$. Moreover, I-LVD in this patient was strikingly higher than the mean values reported by Pak et al. for node-negative cases, $11.26 \pm 3.84$, and N3 cases, 14.16 \pm 5.00 [5].
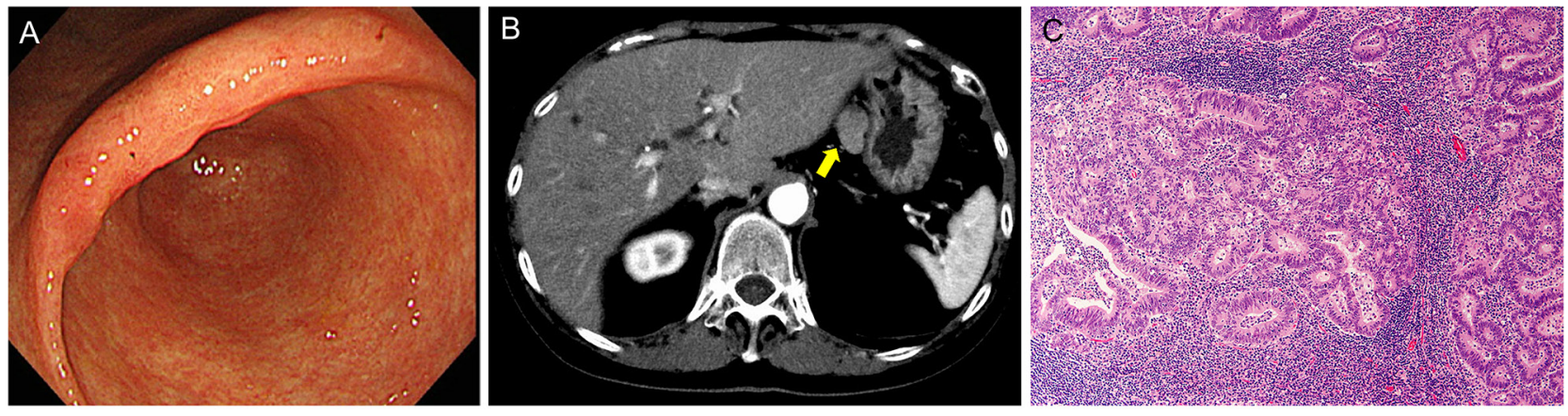

Figure 1: Preoperative findings. (A) Endoscopy showed irregular mucosa in the lesser curvature of the gastric angle with ill-defined margins from surrounding atrophic mucosa. (B) Computed tomography showed an irregular oval tumor of 23 mm diameter beside the left gastric artery (arrow). (C) Laparoscopic biopsy of the intra-abdominal tumor revealed a metastatic tubular adenocarcinoma in the lymph node. Hematoxylin and eosin (H\&E) staining, original magnification, $\times 100$. 


\section{Somatic mutations}

We prepared DNA from microdissected FFPE tissue samples of the primary intramucosal gastric carcinoma, the metastatic LN carcinoma, and a normal tissue, and performed target resequencing using an Ion Proton System
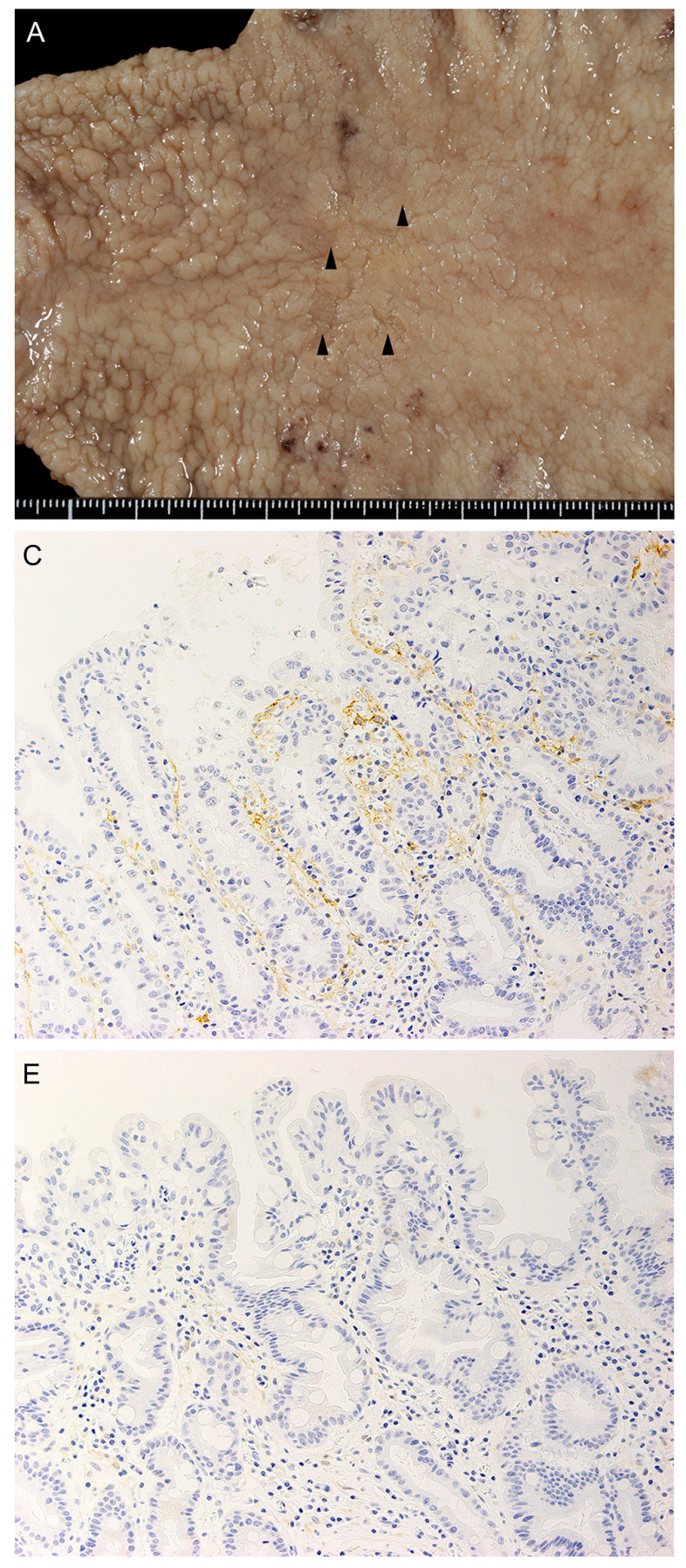

(Thermo Fisher Scientific, Carlsbad, CA, USA). We employed two panels of target genes to cover the known commonly mutated genes in gastric cancer (Table 1) [6-8]. One panel was the IonAmpliSeq ${ }^{\mathrm{TM}}$ Comprehensive Cancer Panel (Thermo Fisher Scientific) that covered the coding exons of 409 cancer-associated genes, and the other was
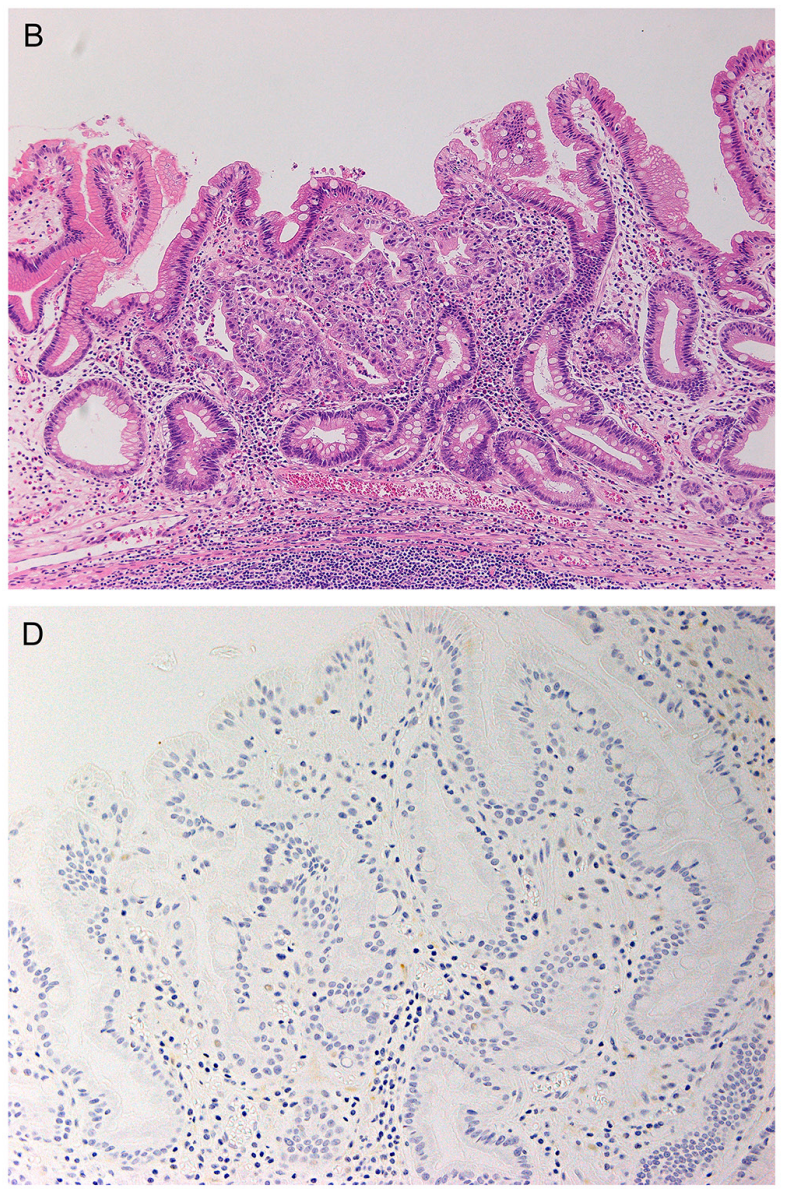

Figure 2: Gross and microscopic pathology of the surgically resected section of the stomach. (A) Four irregular mucosal lesions, each 1-4 $\mathrm{mm}$ in diameter, were located side by side in the lesser curvature (arrowheads). (B) The primary cancer consisted of tubular adenocarcinoma of the well-differentiated type confined within the mucosal layer without ulceration. H\&E staining, original magnification, $\times 100$. (C-E) Images of immunohistochemical staining with anti-D2-40 antibody showed abundant lymphatic vessels in an intratumoral area (C) in contrast with scanty lymphatic vessels in a peritumoral area (D) and a portion of normal mucosa (E). All histological images were taken at the original magnification of $\times 200$. 
Table 1: The list of target genes examined in panel sequencing

\begin{tabular}{|c|c|c|c|c|c|c|c|c|}
\hline$A B L 1$ & CASC5 & $E G F R$ & $G 6 P D$ & KLF6 & $M Y C N$ & PIM1 & $S H 2 D 1 A$ & USP9X \\
\hline$A B L 2$ & $C B L$ & EML4 & GATA1 & $K R A S$ & MYD 88 & PKHD1 & SMAD2 & $V H L$ \\
\hline$A C V R 2 A$ & $C C N D 1$ & EP300 & GATA2 & $L A M P 1$ & MYH11 & PLAG1 & SMAD4 & $W A S$ \\
\hline ADAMTS20 & $C C N D 2$ & EP400 & GATA3 & $L C K$ & МYH9 & PLCG1 & SMARCA4 & WHSC1 \\
\hline$A F F 1$ & CCNE1 & ЕРНАЗ & $G D N F$ & $L I F R$ & $N B N$ & PLEKHG5 & $S M A R C B 1$ & $W R N$ \\
\hline$A F F 3$ & $C D 79 A$ & ЕРНА7 & GNA11 & LPHN3 & NCOA1 & $P M L$ & $S M O$ & WT1 \\
\hline$A K A P 9$ & $C D 79 B$ & EPHB1 & $G N A Q$ & $L P P$ & NCOA2 & PMS1 & SMUG1 & $X P A$ \\
\hline$A K A P 13$ & $C D C 73$ & EPHB4 & GNAS & $L R P 1 B$ & NCOA4 & $P M S 2$ & SOCS1 & $X P C$ \\
\hline$A K T 1$ & $\mathrm{CDH1}$ & ЕРНВ6 & GPR124 & $L T F$ & $N F 1$ & POT1 & $S O X 11$ & XPO1 \\
\hline$A K T 2$ & CDH11 & $E R B B 2$ & $G R M 8$ & $L T K$ & $N F 2$ & POU5F1 & $S O X 2$ & $X R C C 2$ \\
\hline$A K T 3$ & $\mathrm{CDH} 2$ & $E R B B 3$ & $G U C Y 1 A 2$ & $M A F$ & NFE2L2 & $P P A R G$ & $S R C$ & ZNF384 \\
\hline$A L K$ & $\mathrm{CDH} 20$ & $E R B B 4$ & $H C A R 1$ & $M A F B$ & $N F K B 1$ & $P P P 2 R 1 A$ & $S S X 1$ & ZNF521 \\
\hline$A P C$ & $\mathrm{CDH} 5$ & $E R C C 1$ & $H I F 1 A$ & $M A G E A 1$ & $N F K B 2$ & PRDMI & STK11 & \\
\hline$A R$ & $C D K 12$ & $E R C C 2$ & $H L F$ & $M A G I 1$ & $N I N$ & $P R K A R 1 A$ & STK36 & \\
\hline$A R I D I A$ & $C D K 4$ & ERCC3 & $H N F 1 A$ & $M A L T 1$ & $N K X 2-1$ & PRKDC & $S U F U$ & \\
\hline ARID2 & $C D K 6$ & $E R C C 4$ & HOOK3 & $M A M L 2$ & NLRP1 & PSIP1 & $S Y K$ & \\
\hline$A R N T$ & $C D K 8$ & ERCC5 & $H R A S$ & $M A P 2 K 1$ & NOTCH1 & PTCH1 & SYNE1 & \\
\hline$A S X L 1$ & $C D K N 2 A$ & $E R G$ & HSP90AA1 & $M A P 2 K 2$ & NOTCH2 & PTEN & $T A F 1$ & \\
\hline$A T F 1$ & $C D K N 2 B$ & ESR1 & HSP90AB1 & $M A P 2 K 4$ & NOTCH4 & PTGS2 & $T A F 1 L$ & \\
\hline$A T M$ & $C D K N 2 C$ & ETS1 & $I C K$ & $M A P 3 K 7$ & NPM1 & PTPN11 & $T A L 1$ & \\
\hline$A T R$ & $C E B P A$ & ETV1 & $I D H 1$ & $M A P K 1$ & $N R A S$ & PTPRD & $T B X 22$ & \\
\hline$A T R X$ & CHEK1 & ETV4 & IDH2 & $M A P K 8$ & NSD1 & PTPRT & $T C F 12$ & \\
\hline$A U R K A$ & CHEK2 & $E X T 1$ & $I G F 1 R$ & $M A R K 1$ & NTRK1 & RAD50 & $T C F 3$ & \\
\hline$A U R K B$ & $C I C$ & $E X T 2$ & $I G F 2$ & $M A R K 4$ & NTRK3 & $R A F 1$ & TCF7L1 & \\
\hline$A U R K C$ & $C K S 1 B$ & $\mathrm{EZH} 2$ & $I G F 2 R$ & $M B D 1$ & NUMA1 & $R A L G D S$ & TCF7L2 & \\
\hline$A X L$ & $C M P K 1$ & FAM123B & $I K B K B$ & $M C L 1$ & NUP214 & $R A R A$ & TCL1A & \\
\hline$B A I 3$ & COL1A1 & $F A N C A$ & $I K B K E$ & $M D M 2$ & NUP98 & $R B 1$ & TET1 & \\
\hline$B A P 1$ & $C R B N$ & FANCC & $I K Z F 1$ & $M D M 4$ & $P A K 3$ & $R E C Q L 4$ & TET2 & \\
\hline$B C L 10$ & $C R E B 1$ & FANCD2 & $I L 2$ & MEN1 & $P A L B 2$ & $R E L$ & TFE3 & \\
\hline$B C L 11 A$ & $C R E B B P$ & $F A N C F$ & $I L 21 R$ & $M E T$ & PARP1 & $R E T$ & $T G F B R 2$ & \\
\hline$B C L 11 B$ & $C R K L$ & $F A N C G$ & $I L 6 S T$ & MITF & $P A X 3$ & RHOA & $T G M 7$ & \\
\hline$B C L 2$ & CRTC1 & $F A S$ & $I L 7 R$ & MLH1 & $P A X 5$ & RHOH & THBS1 & \\
\hline$B C L 2 L 1$ & CSF1R & $F B X W 7$ & ING4 & $M L L$ & $P A X 7$ & $R N A S E L$ & TIMP3 & \\
\hline$B C L 2 L 2$ & CSMD3 & FGFR1 & IRF4 & MLL2 & $P A X 8$ & $R N F 2$ & $T L R 4$ & \\
\hline$B C L 3$ & CTNNA1 & $F G F R 2$ & $I R S 2$ & MLL3 & PBRMI & $R N F 213$ & $T L X 1$ & \\
\hline$B C L 6$ & CTNNB1 & FGFR3 & ITGA10 & MLLT10 & $P B X 1$ & ROS1 & TNFAIP3 & \\
\hline$B C L 9$ & $C Y L D$ & FGFR4 & ITGA9 & $M M P 2$ & PDE4DIP & RPS6KA2 & TNFRSF 14 & \\
\hline$B C R$ & СYР2C19 & $\mathrm{FH}$ & $I T G B 2$ & MN1 & $P D G F B$ & $R R M 1$ & $T N K 2$ & \\
\hline$B I R C 2$ & $C Y P 2 D 6$ & $F L C N$ & $I T G B 3$ & $M P L$ & PDGFRA & $R U N X 1$ & TOP1 & \\
\hline
\end{tabular}

(Continued) 


\begin{tabular}{|c|c|c|c|c|c|c|c|}
\hline$B I R C 3$ & $D A X X$ & FLII & $J A K 1$ & MRE11A & PDGFRB & RUNX1T1 & TP53 \\
\hline BIRC5 & $D C C$ & FLT1 & $J A K 2$ & MSH2 & PERI & SAMD9 & $T P R$ \\
\hline$B L M$ & $D D B 2$ & FLT3 & $J A K 3$ & MSH6 & $P G A P 3$ & $S B D S$ & TRIM24 \\
\hline$B L N K$ & DDIT3 & FLT4 & $J U N$ & MTOR & PHOX2B & SDHA & TRIM33 \\
\hline BMPRIA & $D D R 2$ & FN1 & KAT6A & MTR & PIK $3 C 2 B$ & $S D H B$ & TRIP11 \\
\hline$B R A F$ & $D E K$ & FOXL2 & KАT6B & MTRR & PIK $3 C A$ & SDHC & TRRAP \\
\hline$B R D 3$ & DICER1 & FOXO1 & KDM5C & MUC1 & PIK3CB & $S D H D$ & TSC1 \\
\hline BRIP1 & $D L C 1$ & FOXO3 & KDM6A & MUTYH & PIK $3 C D$ & SEPTP & TSC2 \\
\hline BTK & DNMT3A & FOXP1 & $K D R$ & $M Y B$ & PIK3CG & SETD2 & TSHR \\
\hline$B U B 1 B$ & $D P Y D$ & FOXP4 & $K E A P 1$ & $M Y C$ & PIK3R1 & $S F 3 B 1$ & UBR5 \\
\hline CARD11 & $D S T$ & FZR1 & KIT & MYCL1 & PIK3R2 & $S G K 1$ & UGT1A1 \\
\hline
\end{tabular}

an Ion AmpliSeq ${ }^{\mathrm{TM}}$ Custom DNA Panel (Thermo Fisher Scientific) that was designed to cover the coding exons of RHOA and its regulatory molecules, $A K A P 13$ and $D L C 1$ (Table 1). The mean coverage of each panel was 241.4 and 4816.3 fold per amplicon, respectively. We identified somatic mutations in $N B N$, p.P6S, and $P A X 8$, p.R $49 \mathrm{H}$, in the LN metastasis; however, we did not identify these mutations in the primary tumor (Table 2). These mutations were confirmed by Sanger sequencing (Figure 3A, 3B).

\section{Functional analysis of $P A X 8$, p.R49H}

To determine a functional phenotype of the $P A X 8^{\mathrm{R} 49 \mathrm{H}}$, we compared transactivation activities between the wild type $P A X 8$ and the mutant $P A X 8$, i.e., $P A X 8^{\mathrm{R} 49 \mathrm{H}}$, toward $E 2 F 1$ that was known to be a transcriptional target of paired box 8 (PAX8) [9]. We constructed expression vectors harboring the wild type $P A X 8$ or the mutant $P A X 8$, and transfected them into $293 \mathrm{~T}$ cells. The transfection induced an equivalent level of exogenous expression of encoded proteins as indicated by immunoblots in Figure 4A. Then, we measured transcriptions of $E 2 F 1$ in the cells by the quantitative real time PCR assay. The result showed that the mutant PAX8 induced a significantly increased level of transcription of E2F1 compared to the wild-type PAX8 as shown in Figure 4B $(\mathrm{P}<0.001)$. These results indicate that the $P A X 8^{\mathrm{R} 49 \mathrm{H}}$ may exhibit a gain-of-function phenotype compared to the wild-type PAX8.

\section{DISCUSSION}

Taking a molecular pathologic approach, we examined a peculiar case of gastric intramucosal adenocarcinoma with LN metastasis. The primary tumor seemed to fulfill the criteria for the absolute indication of ER; however, because of the LN metastasis, we performed a surgical resection with lymphadenectomy. In pathological examination, the primary tumor was accompanied by dense lymphatic vessels, and I-LVD proved to be particularly high. This finding suggests that the elevated level of lymphangiogenesis accompanying the adenocarcinoma could have increased the chances of primary cancer cells intravasating lymphatic vessels. LVD is known to be associated with $\mathrm{LN}$ metastasis in various human cancers [10]. As for gastric cancer, although differences in the pathological roles of I-LVD and P-LVD are still controversial, several reports showed that I-LVD was higher in tumors associated with LN metastasis $[5,11$, 12]. To the best of our knowledge, our case is the first to show that high I-LVD may facilitate LN metastasis even in a case of an intramucosal gastric carcinoma that seemed to meet the criteria for the absolute indication of ER. In the present case, the gastric cancer and metastatic LN were surgically resected because the swollen LN detected by CT was proven to be a metastasis by laparoscopic biopsy before surgery. This demonstrates that careful evaluation of LN state using CT is important, as was previously reported [4]. When LN metastasis is not identifiable on CT, I-LVD could potentially be measured to assess the risk of metastasis after ER because I-LVD can be evaluated in the ER specimen.

Next, we investigated the coding exons of 412 cancer-associated genes by next-generation sequencing. As the primary cancer was uncommonly multicentric localized adjacent to each other, which suggests the possibility of a pre-existing mutational accumulation in the atrophic gastric mucosa due to a history of $H$. pylori infection as previously reported [13], we avoided using noncancerous mucosa as a normal control sample for this analysis. The results indicated that the present prometastatic intramucosal gastric cancer with extraordinary LN metastasis did not harbor any common mutations for gastric cancer such as TP53, ARIDIA, PIK3CA, CDH1, SMAD4, APC, KRAS, or RHOA or its regulatory molecules, $A K A P 13$ and $D L C 1$. Instead, the present case harbored somatic mutations in the LN metastasis: $N B N$, p.P6S, and PAX8, p.R49H. 
Table 2: Annotations for somatic mutations of $N B N$ and $P A X 8$

\begin{tabular}{|c|c|c|c|c|c|c|c|c|c|c|c|}
\hline Gene & Position & Exon & $\begin{array}{c}\text { Coding } \\
\text { DNA }\end{array}$ & $\begin{array}{c}\text { Amino } \\
\text { acid }\end{array}$ & $\begin{array}{c}\text { COSMIC } \\
\text { ID }\end{array}$ & SIFT & $\begin{array}{c}\text { Polyphen2 } \\
\text { HDIV }\end{array}$ & $\begin{array}{c}\text { Mutation } \\
\text { Taster }\end{array}$ & GQ & DP & $\mathbf{A F}$ \\
\hline$N B N$ & Chr8:90996774 & 1 & c. $16 \mathrm{C}>\mathrm{T}$ & p.P6S & 1102345 & $\begin{array}{l}\text { tolerated } \\
\quad(0.13)\end{array}$ & $\begin{array}{l}\text { benign } \\
(0.429)\end{array}$ & $\begin{array}{l}\text { disease } \\
\text { causing }\end{array}$ & 58 & 122 & 0.11 \\
\hline$P A X 8$ & Chr2:114004376 & 3 & c. $146 \mathrm{G}>\mathrm{A}$ & p.R49H & - & $\begin{array}{c}\text { damaging } \\
(0)\end{array}$ & $\begin{array}{c}\text { Probably } \\
\text { damaging } \\
(0.999)\end{array}$ & $\begin{array}{l}\text { disease } \\
\text { causing }\end{array}$ & 114 & 179 & 0.14 \\
\hline
\end{tabular}

$\mathrm{AF}$, allele frequency based on flow evaluator observation counts; DP, total read depth at the locus; GQ, genotype quality.

A

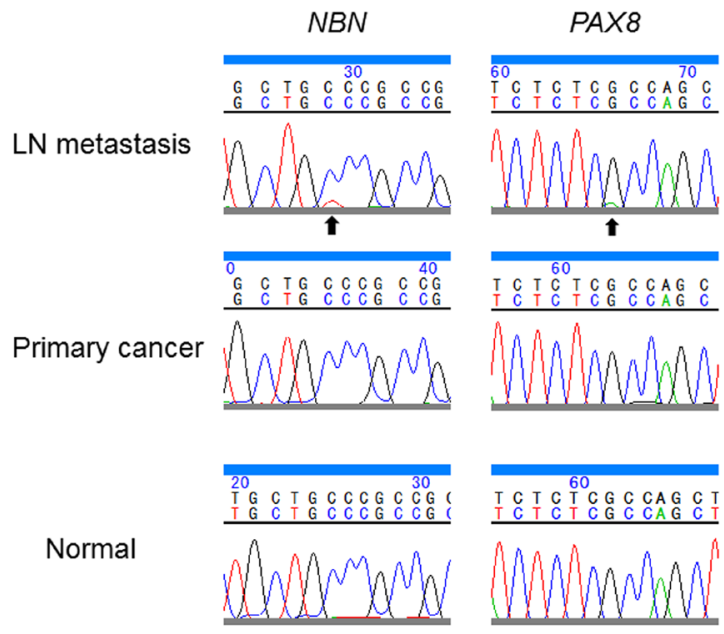

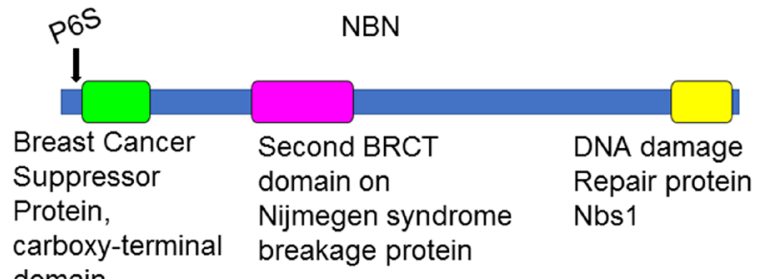

domain

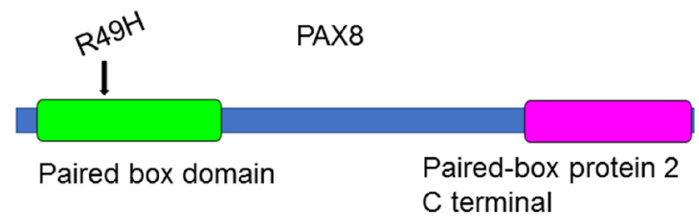

Figure 3: Somatic mutations in $N B N$ and $P A X 8$ identified in the present case. (A) Validation by Sanger sequencing showed single nucleotide substitution in $N B N$ and $P A X 8$ in the lymph node metastasis (arrow) but not in the primary tumor. (B) Functional domains (colored boxes) and mutated residues (arrows) in nibrin (NBN) and paired box 8 (PAX8).

A

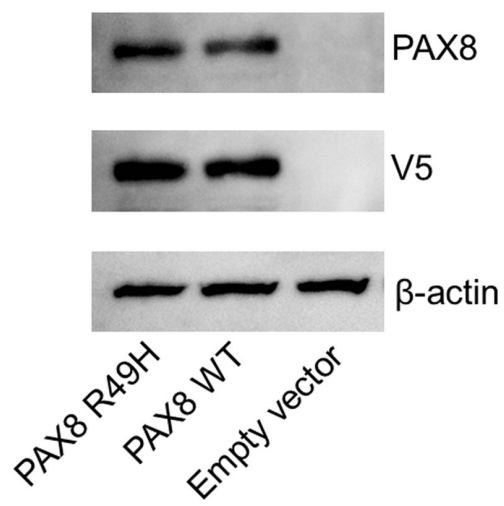

B

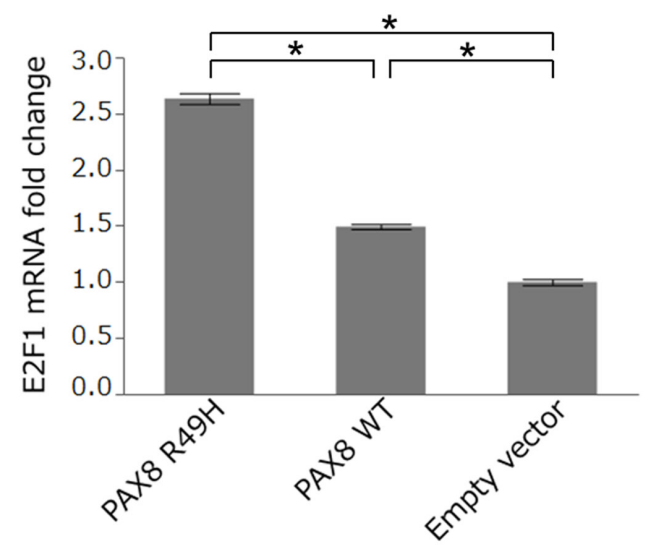

Figure 4: Transactivation of $\boldsymbol{E} 2 \boldsymbol{F} 1$ by $\boldsymbol{P A X 8}$. (A) Immunoblot of $293 \mathrm{~T}$ cells transfected with the $P A X 8^{\mathrm{R} 49 \mathrm{H}}-\mathrm{V} 5-\mathrm{His}$ (PAX8 R49H),

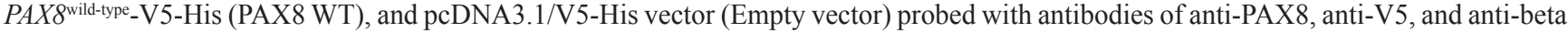
actin. (B) The relative expression of E2F1 in each transfected 293T cells measured by the quantitative real-time PCR assay. The expression of $E 2 F 1$ was normalized to the expression of $G A P D H$ and analyzed by means of the $2^{-\triangle A C T}$ method. An asterisk indicated $\mathrm{P}<0.001$. 
The identification of only a few mutated genes could be explained by the early stage of the cancer. Molecular pathologic information of such an early-stage cancer with an aberrant prometastatic nature can be a valuable source to help elucidate the mechanism of metastasis because analyzing such cases may lead to the identification of molecular alterations associated with metastasis in a small number of mutated genes.

$N B N$ encodes nibrin (NBN), a member of the MRE11/RAD50 double-strand break repair complex [14]. A truncating mutation in $N B N$ causes a defective response to DNA double-strand breaks, which results in an unstable genome and a predisposition to malignancies [15]. The mutated P6 residue of $N B N$ is not located within any functional domains. However, the P6S mutation of $N B N$ was identified in a patient with uterine corpus endometrioid carcinoma (COSM1102345 in the COSMIC database, http://cancer.sanger.ac.uk/cosmic). $N B N$, p.K653fs is reported to be identified in peritoneal metastasis of gastric cancer by whole-exome sequencing elsewhere [16]. Although functional impacts of $N B N^{\mathrm{P} 6 \mathrm{~S}}$ are not known, the mutation could cause genomic instability or copy number variations.

PAX8 encodes paired box 8 (PAX8), a transcription factor required for the formation of thyroxine-producing follicular cells, of endodermal origin [17]. $P A X 8^{\mathrm{R} 49 \mathrm{C}}$ has been identified as a somatic mutation in gastric cancer according to the COSMIC database (COSM4084322); however, $P A X 8^{\mathrm{R} 49 \mathrm{H}}$ has not been reported. The R49 residue of $P A X 8$ is located within the paired box domain (Figure 3B), which may be the reason that $P A X 8^{\mathrm{R} 49 \mathrm{H}}$ was predicted to be functionally damaging by some prediction programs, namely Polyphen-2 (http:// genetics.bwh.harvard.edu/pph2/), SIFT (http://sift.jcvi. org/), and MutationTaster (http://www.mutationtaster. org/). Missense mutations within the paired box domain, such as p.Q40P, p.S54G, p.C57Y, p.L62R, are known to cause congenital hypothyroidism, thyroid hypoplasia and aplasia, and/or kidney agenesis due to the loss of its transactivation effect [18-21]. PAX8 is expressed highly in the thyroid and kidney as well as slightly but evidently in gastric mucosa and gastric cancer (GEO10420251 and GEO95672775 in Gene Expression Omnibus Profiles, https://www.ncbi.nlm.nih.gov/geoprofiles). PAX8 is reported to be expressed in metastatic non-small cell lung cancers and to promote cell migration via interaction with MET and RON [22].

By our experiments testing a transactivating function of $P A X 8$ toward $E 2 F 1$, we found that the $P A X 8^{\mathrm{R} 49 \mathrm{H}}$ was able to show a gain-of-function phenotype in transactivation of $E 2 F 1$ compared to the wild type $P A X 8$. E2F transcription factor 1 (E2F1) encoded by $E 2 F 1$ is well known for its tumor suppressive role in conjunction with retinoblastoma protein 1 (RB1), however, it is also known to play some promoting roles in cancer as reported to promote an epithelial- mesenchymal transition (EMT) by transactivating FOXL2 in gastric cancer cells, which may be associated with increased LN metastasis in patients with gastric cancer [23]. Thus, our result and these compelling evidences suggest that $P A X 8^{\mathrm{R} 49 \mathrm{H}}$ could have promoted EMT and subsequent LN metastasis via E2F1 in the present case.

In the present case, the somatic mutations were identified only in the metastatic LN tissue and not in the primary tumor. To explain this crucial observation, one can argue that the LN metastasis was not derived from the gastric adenocarcinoma but from elsewhere in other organs. However, we determined that the LN tumor was likely to be a metastasis of the intramucosal gastric carcinoma, which was cured by the surgical resection, through the following evidence: identical histology between the gastric tumor and the metastatic LN tumor, anatomical location of the metastatic LN, and the concerted decrease of elevated tumor markers. Moreover, the patient has not manifested any other malignant neoplasms for more than 3 years after surgery and no adjuvant chemotherapy. Alternatively, the failure of finding mutations in the intramucosal gastric carcinoma could be due to tumor heterogeneity, i.e., a small number of prometastatic clones in the primary tumor. A previous report indicated that a lethal metastatic clone of prostate cancer was derived from only a single small lesion in 36 sectioned blocks of the primary cancer [24]. Thus, it is likely that cells with a metastatic ability may have existed as just a tiny fraction in the primary tumor, which could not be detected. On the other hand, we could not exclude the possibility that the mutations occurred after metastasizing to the LN. Postmetastatic mutations could give advantages of survival and growth of cells in the metastatic site. Fractions of mutated calls were $11 \%$ and $14 \%$ in $N B N$ and $P A X 8$, respectively. This relatively minor fraction of mutated alleles may be due to 1) heterogeneity of cancer cells, 2) wild cancer cells derived from the collective dissemination of tumor clusters [25], or 3) normal lymphocytes with density drastically higher than that of the cancer cells, as shown in Figure 1C, meaning the ratio of cancer-derived DNA should be substantially lowered by contamination of even a small volume of peripheral lymphocytes. This could encourage the assumption that these mutations contributed to facilitation and/or development of LN metastasis, since they are more selected in the metastatic LN.

In conclusion, intramucosal gastric carcinoma that seemed to fulfill the criteria for the absolute indication of ER had a LN metastasis and thus was resected surgically. Increased lymphangiogenesis was observed in the primary tumor. Moreover, somatic mutations of $N B N$, p.P6S, and $P A X 8$, p.R $49 \mathrm{H}$ were observed in the metastatic tumor. The $P A X 8^{\mathrm{R} 49 \mathrm{H}}$ showed a gain-offunction phenotype in transactivation of E2F1. These 
findings may serve not only to develop biomarkers and/or molecular therapeutic targets but also to revise current recommendation for ER resection of early gastric cancer.

\section{MATERIALS AND METHODS}

\section{Ethics and informed consent}

This study was approved by the ethical committee of Tokyo Women's Medical University (protocol \#272). Written informed consent was obtained from the patient for research and publication.

\section{Quantitative analysis of lymphatic vessel density}

FFPE tissue sections were immunohistochemically stained by using anti-D2-40 antibody (Covance Antibody Products, San Diego, CA, USA) and Autostainer Link 48 (Dako, Glostrup, Denmark). Quantitative analysis of lymphatic vessel density (LVD) was performed by counting the D2-40 stained lymphatic vessels according to Pak et al [5]. Intratumoral (I)-LVD, Peritumoral (P)-LVD, and LVD in the normal control tissues (C-LVD) were counted.

\section{Panel design for the next-generation sequencing}

The Ion AmpliSeq ${ }^{\mathrm{TM}}$ Comprehensive Cancer Panel covering all coding exons of 409 cancer-associated genes and an Ion AmpliSeq ${ }^{\mathrm{TM}}$ Custom DNA Panel covering all coding exons of $R H O A$ and its regulatory molecules, $A K A P 13$ and $D L C 1$, were used. In total, all coding exons of 412 genes were examined (Table 1).

\section{Tissue dissection and DNA preparation}

FFPE tissue samples from the primary gastric tumor, the metastatic LN tumor, and a normal portion of the stomach (submucosa or deeper area) were used for genetic analysis. Areas of adenocarcinoma found by microscopic observation were manually dissected. DNA was prepared with a QIAamp DNA FFPE Tissue Kit (Qiagen, Hilden, Germany). DNA from the metastatic LN tumor and normal gastric tissue were analyzed by next-generation sequencing. For the primary cancer, since each lesion was small, we mixed the DNA together and only analyzed it by Sanger sequencing.

\section{Next-generation sequencing}

Sequencing libraries were prepared using Ion AmpliSeq ${ }^{\text {TM }}$ Library Kit 2.0 (Thermo Fisher Scientific) according to the manufacturer's instruction. The quantity of DNA amplicons was evaluated using a High Sensitivity DNA kit (Agilent Technologies, Waldbronn, Germany). Sequencing was performed using an Ion Proton ${ }^{\text {TM }}$ System (Thermo Fisher Scientific) according to the manufacturer's instructions.

\section{Variant calling and annotation}

Data analyses were performed using the Ion Torrent Suite Software (version 5.0.3). After base calling, the reads were aligned against the reference human genome (hg19) using the TMAP algorithm within the Torrent Suite. Variants with Genome quality $>50$ and an allele frequency $>10 \%$ were considered. For further single nucleotide polymorphism (SNP) analysis, only non-synonymous nucleotide exchanges were considered. SNPs reported to be $>1 \%$ in 1000 G, ExAC or ESP6500si were dismissed. SNPs detected only in tumor tissues were counted. All somatic variations annotated were validated by Sanger sequencing.

\section{Sanger sequencing}

Genomic portions of somatic mutations were amplified by using paired primers of 5'-GGTTACGCGGTTGCACGTCG-3' and 5'-TCTGCCC TTACCTCCTGCCG-3' for $N B N$ and $5^{\prime}$-CTTTGTGAA TGGCAGACCTC-3' and 5'-AAGGATCTTGCTGACGCA GC-3' for $P A X 8$. The amplified products were analyzed by Sanger sequencing, as described previously [26].

\section{Cell culture}

The human embryonic kidney 293T was obtained from the European Collection of Authenticated Cell Cultures (ECACC 12022001). The cells were cultured using Dulbecco's Modified Eagle's Medium (Sigma, St. Louis, MO, USA) with $10 \%$ fetal bovine serum in a humidified incubator at $37^{\circ} \mathrm{C}$ with $5 \% \mathrm{CO}_{2}$.

\section{Plasmid vectors}

The wild-type $P A X 8$ cDNA was amplified from a fetal kidney cDNA library (Agilent Technologies) by PCR using the KOD Plus DNA polymerase system (TOYOBO, Osaka, Japan). The paired primers used were as follows: forward 5'-TTTAAGCTT/ CCCCGGCGATGCCTCACAAC-3', and reverse 5'-TTTGAATTC/CAGATGGTCAAAGGCCGTGGC-3'. The amplified product was separated by agarose gel electrophoresis. A band corresponding to an equivalent molecular weight of PAX8 (NM_003466.3) was extracted, purified, and cloned into the pcDNA3.1/V5-His vector (Invitrogen, San Diego, CA, USA) at the HindIII and EcoRI sites to generate the wild-type PAX8-V5-His vector. The mutant $P A X 8$ (p.R49H)-V5-His vector was generated by means of a site-directed mutagenesis technique using QuikChange II Site-Directed Mutagenesis Kit (Agilent Technologies) with following primers: 5'GACGCGGAGCTGGTGAGAGATGTCGCAG-3' and 5'-CTGCGACATCTCTCACCAGCTCCGCGTC-3'

according to the manufacture's instruction. Nucleotide sequences of the created plasmid vectors were confirmed by Sanger sequencing. 


\section{Cell transfection}

293T cells were seeded at a concentration of $5 \times 10^{5}$ cells/well in 6-well plates $24 \mathrm{~h}$ before transfection. Transfection of each created plasmid vectors or the control pcDNA3.1/V5-His vector was performed using Lipofectamine 2000 Transfection Reagent (Thermo Fisher Scientific) according to the manufacturer's instruction. 24 $\mathrm{h}$ after transfection, cells were collected and proceeded to following immunoblotting and the quantitative real-time PCR assay. The experiment was performed twice in duplicate.

\section{Immunoblotting}

Collected cells were lysed in modified RIPA buffer containing $1 \times$ complete mini protease inhibitor cocktail (Sigma) and $1 \times$ PhosSTOP phosphatase inhibitor cocktail (Sigma). Cell extracts containing equal amounts of proteins were boiled in loading buffer, applied to 10$20 \%$ polyacrylamide gradient gel, and separated by electrophoresis. Then the proteins were blotted onto a polyvinylidene difluoride membrane (ATTO, Tokyo, Japan). After blocking using the ECL Blocking Agent (Amersham Biosciences, Buckinghamshire, UK) for $1 \mathrm{~h}$, the membrane was incubated with primary antibodies overnight. Primary antibodies used were the mouse monoclonal anti-PAX8 antibody (1:200 dilution) (Santa Cruz Biotechnology, Dallas, TX, USA), the mouse monoclonal anti-V5 antibody (1:5000 dilution) (Thermo Fisher Scientific), and the mouse monoclonal anti- $\beta$ actin antibody (1:1000 dilution) (Sigma). The membrane was incubated with a secondary antibody for $1 \mathrm{~h}$. The secondary antibody used was horseradish peroxidaseconjugated anti-mouse immunoglobulin antibody (1:10000 dilution) (GE Healthcare, Buckinghamshire, UK). Signals were visualized using the ECL Prime Western Blotting Detection Reagent (Amersham Biosciences) and LAS 4000 Mini system (Fujifilm, Tokyo, Japan).

\section{Quantitative real-time PCR assay}

Total RNA was isolated from collected cells using RNeasy Mini kit (Qiagen, Hilden, Germany). cDNA synthesis was performed using High-Capacity cDNA Reverse Transcription Kit (Thermo Fisher Scientific). Pre-designed primer/probe sets for human PAX8 (Hs00247586_ml) and E2F1 (Hs00153451_ml) in TaqMan gene expression assay system (Thermo Fisher Scientific) were used in the quantitative real-time PCR assay. GAPDH was used as an endogenous control. The analyses were performed by means of the $2^{-\Delta \Delta C T}$ method [27] upon the 7500 Real-Time PCR system (Thermo Fisher Scientific) according to the manufacture's instruction. The experiment was performed twice in triplicate.

\section{Statistical analyses}

Continuous data are described as the mean and standard deviation and were compared using Tukey's method.

\section{Abbreviations}

CA19-9, carbohydrate antigen 19-9; CEA, carcinoembryonic antigen; C-LVD, Lymphatic vessel density in the normal control tissues; CT, computed tomography; EMT, epithelial-mesenchymal transition; ER, endoscopic resection; FFPE, Formalin-fixed and paraffin-embedded; $\mathrm{H} \& \mathrm{E}$, hematoxylin and eosin; I-LVD, Intratumoral-lymphatic vessel density; LN, lymph node; LVD, lymphatic vessel density; NBN, nibrin; PAX8, paired box 8; PCR, polymerase chain reaction; P-LVD, Peritumoral lymphatic vessel density; $\mathrm{RB} 1$, retinoblastoma protein 1 .

\section{Author contributions}

NI, SA, MY, SM, and TF conceived the study and designed the experiments. KT coordinated sample acquisition. NI, SA, KT, TY, and MY contributed in acquisition of clinical data. NI, ET, and TM performed experiments. YS, NI, SY, TM, and TF performed the bioinformatics data analysis. NI, SA, MY, SM, and TF contributed to drafting and critical review of manuscript.

\section{ACKNOWLEDGMENTS}

We are grateful to the patient whose commitment to the research and tissue donation made this study possible.

\section{CONFLICTS OF INTEREST}

The authors declare no conflicts of interest.

\section{FUNDING}

This work was supported by JSPS KAKENHI Grant Number JP16K10518.

\section{REFERENCES}

1. Japanese gastric cancer association. Japanese gastric cancer treatment guidelines 2014 (ver. 4). Gastric Cancer. 2017; 20:1-19.

2. Lee S, Choi KD, Hong SM, Park SH, Gong EJ, Na HK, Ahn JY, Jung KW, Lee JH, Kim DH, Song HJ, Lee GH, Jung HY, Kim JH. Pattern of extragastric recurrence and the role of abdominal computed tomography in surveillance after endoscopic resection of early gastric cancer: korean experiences. Gastric Cancer. 2017; 20:843-52. https://doi. org/10.1007/s10120-017-0691-z. 
3. Tanabe S, Ishido K, Matsumoto T, Kosaka T, Oda I, Suzuki H, Fujisaki J, Ono H, Kawata N, Oyama T, Takahashi A, Doyama $\mathrm{H}$, Kobayashi M, et al. Long-term outcomes of endoscopic submucosal dissection for early gastric cancer: a multicenter collaborative study. Gastric Cancer. 2017; 20:45-52.

4. Kim DJ, Kim W. A case of single lymph node metastasis near the common hepatic artery following a curative endoscopic resection for gastric mucosal cancer. Gastric Cancer. 2014; 17:387-391.

5. Pak KH, Jo A, Choi HJ, Choi Y, Kim H, Cheong JH. The different role of intratumoral and peritumoral lymphangiogenesis in gastric cancer progression and prognosis. BMC Cancer. 2015; 15:498.

6. Bass AJ, Thorsson V, Shmulevich I, Reynolds SM, Miller M, Bernard B, Hinoue T, Laird PW, Curtis C, Shen H, Weisenberger DJ, Schultz N, Shen R, et al, and Cancer Genome Atlas Research Network. Comprehensive molecular characterization of gastric adenocarcinoma. Nature. 2014; 513:202-209.

7. Kakiuchi M, Nishizawa T, Ueda H, Gotoh K, Tanaka A, Hayashi A, Yamamoto S, Tatsuno K, Katoh H, Watanabe Y, Ichimura T, Ushiku T, Funahashi S, et al. Recurrent gain-of-function mutations of RHOA in diffuse-type gastric carcinoma. Nat Genet. 2014; 46:5837.

8. Ushiku T, Ishikawa S, Kakiuchi M, Tanaka A, Katoh H, Aburatani H, Lauwers GY, Fukayama M. RHOA mutation in diffuse-type gastric cancer: a comparative clinicopathology analysis of 87 cases. Gastric Cancer. 2016; 19:403-411.

9. Li CG, Nyman JE, Braithwaite AW, Eccles MR. PAX8 promotes tumor cell growth by transcriptionally regulating E2F1 and stabilizing RB protein. Oncogene. 2011; 30:4824-4834.

10. Karaman S, Detmar M. Mechanisms of lymphatic metastasis. J Clin Invest. 2014; 124:922-928.

11. Ikeda K, Oki E, Saeki H, Ando K, Morita M, Oda Y, Imamura M, Kakeji Y, Maehara Y. Intratumoral lymphangiogenesis and prognostic significance of VEGFC expression in gastric cancer. Anticancer Res. 2014; 34:3911-3915.

12. Wang XL, Fang JP, Tang RY, Chen XM. Different significance between intratumoral and peritumoral lymphatic vessel density in gastric cancer: a retrospective study of 123 cases. BMC Cancer. 2010; 10:299.

13. Shimizu T, Marusawa H, Matsumoto Y, Inuzuka T, Ikeda A, Fujii Y, Minamiguchi S, Miyamoto S, Kou T, Sakai Y, Crabtree JE, Chiba T. Accumulation of somatic mutations in TP53 in gastric epithelium with Helicobacter pylori infection. Gastroenterology. 2014; 147:407-417.

14. Carney JP, Maser RS, Olivares H, Davis EM, Le Beau M, Yates JR, Hays L, Morgan WF, Petrini JH. The hMre11/ hRad50 protein complex and Nijmegen breakage syndrome: linkage of double-strand break repair to the cellular DNA damage response. Cell. 1998; 93:477-486.

15. Chrzanowska KH, Gregorek H, Dembowska-Bagińska B, Kalina MA, Digweed M. Nijmegen breakage syndrome (NBS). Orphanet J Rare Dis. 2012; 7:13.
16. Liu H, Li F, Zhu Y, Li T, Huang H, Lin T, Hu Y, Qi X, Yu J, Li G. Whole-exome sequencing to identify somatic mutations in peritoneal metastatic gastric adenocarcinoma: a preliminary study. Oncotarget. 2016; 7:43894-906. https://doi.org/10.18632/oncotarget.9707.

17. Mansouri A, St-Onge L, Gruss P. Role of Pax genes in endoderm-derived organs. Trends Endocrinol Metab. 1999; 10:164-167.

18. Macchia PE, Lapi P, Krude H, Pirro MT, Missero C, Chiovato L, Souabni A, Baserga M, Tassi V, Pinchera A, Fenzi G, Grüters A, Busslinger M, Di Lauro R. PAX8 mutations associated with congenital hypothyroidism caused by thyroid dysgenesis. Nat Genet. 1998; 19:83-86.

19. Vilain C, Rydlewski C, Duprez L, Heinrichs C, Abramowicz M, Malvaux P, Renneboog B, Parma J, Costagliola S, Vassart G. Autosomal dominant transmission of congenital thyroid hypoplasia due to loss-of-function mutation of $P A X 8$. J Clin Endocrinol Metab. 2001; 86:234-238.

20. Meeus L, Gilbert B, Rydlewski C, Parma J, Roussie AL, Abramowicz M, Vilain C, Christophe D, Costagliola S, Vassart G. Characterization of a novel loss of function mutation of $P A X 8$ in a familial case of congenital hypothyroidism with in-place, normal-sized thyroid. J Clin Endocrinol Metab. 2004; 89:4285-4291.

21. Congdon T, Nguyen LQ, Nogueira CR, Habiby RL, Medeiros-Neto G, Kopp P. A novel. Mutation (Q40P) in $P A X 8$ associated with congenital hypothyroidism and thyroid hypoplasia: evidence for phenotypic variability in mother and child. J Clin Endocrinol Metab. 2001; 86:3962-3967.

22. Kanteti R, El-Hashani E, Dhanasingh I, Tretiakova M, Husain AN, Sharma S, Sharma J, Vokes EE, Salgia R. Role of PAX8 in the regulation of MET and RON receptor tyrosine kinases in non-small cell lung cancer. BMC Cancer. 2014; 14:185.

23. Dong J, Wang R, Ren G, Li X, Wang J, Sun Y, Liang J, Nie Y, Wu K, Feng B, Shang Y, Fan D. HMGA2-FOXL2 axis regulates metastases and epithelial-to-mesenchymal transition of chemoresistant gastric cancer. Clin Cancer Res. 2017; 23:3461-3473.

24. Haffner MC, Mosbruger T, Esopi DM, Fedor H, Heaphy CM, Walker DA, Adejola N, Gürel M, Hicks J, Meeker AK, Halushka MK, Simons JW, Isaacs WB, et al. Tracking the clonal origin of lethal prostate cancer. J Clin Invest. 2013; 123:4918-4922.

25. Lambert AW, Pattabiraman DR, Weinberg RA. Emerging biological principles of metastasis. Cell. 2017; 168:670-691.

26. Kuboki Y, Shimizu K, Hatori T, Yamamoto M, Shibata N, Shiratori K, Furukawa T. Molecular biomarkers for progression of intraductal papillary mucinous neoplasm of the pancreas. Pancreas. 2015; 44:227-35.

27. Livak KJ, Schmittgen TD. Analysis of relative gene expression data using real-time quantitative PCR and the 2(-Delta Delta C(T)) method. Methods. 2001; 25:402-08. 